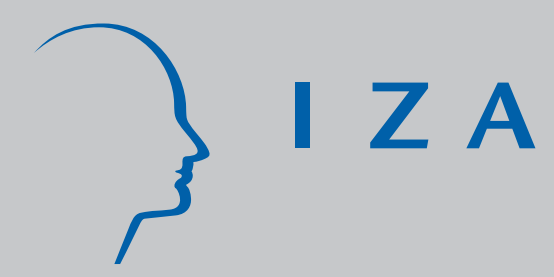

IZA DP No. 2592

Studying J ustice: Measurement, Estimation, and Analysis of the Actual Reward and the J ust Reward

Guillermina J asso

J anuary 2007 


\title{
Studying Justice: Measurement, Estimation, and Analysis of the Actual Reward and the Just Reward
}

\author{
Guillermina Jasso \\ New York University and IZA
}

Discussion Paper No. 2592

January 2007

\author{
IZA \\ P.O. Box 7240 \\ 53072 Bonn \\ Germany \\ Phone: +49-228-3894-0 \\ Fax: +49-228-3894-180 \\ E-mail: iza@iza.org
}

\begin{abstract}
Any opinions expressed here are those of the author(s) and not those of the institute. Research disseminated by IZA may include views on policy, but the institute itself takes no institutional policy positions.

The Institute for the Study of Labor (IZA) in Bonn is a local and virtual international research center and a place of communication between science, politics and business. IZA is an independent nonprofit company supported by Deutsche Post World Net. The center is associated with the University of Bonn and offers a stimulating research environment through its research networks, research support, and visitors and doctoral programs. IZA engages in (i) original and internationally competitive research in all fields of labor economics, (ii) development of policy concepts, and (iii) dissemination of research results and concepts to the interested public.
\end{abstract}

IZA Discussion Papers often represent preliminary work and are circulated to encourage discussion. Citation of such a paper should account for its provisional character. A revised version may be available directly from the author. 


\section{ABSTRACT \\ Studying Justice: Measurement, Estimation, and Analysis of the Actual Reward and the Just Reward ${ }^{*}$}

This paper describes procedures for measuring and estimating the fundamental quantities in the study of distributive justice. We examine a variety of methods for measuring the actual reward and the just reward, for both self and other, including direct and indirect methods for measuring the just reward. Finally, we provide an extended illustration of one of the two indirect methods, the one-reward-per-rewardee method, obtaining estimates not only of ideas of the just earnings for others but also of just rates of return to personal characteristics as well as perceived overall injustice and its decomposition into poverty and inequality components.

JEL Classification: D1, D31, D6, D8, I3, J31

Keywords: fairness, occupations, Rossi's factorial survey method, vignettes, experienced and expressed justice evaluations, justice evaluation function, loss aversion, Golden Number, Gini index, Atkinson's inequality measure, Theil's MLD, justice index

Corresponding author:

Guillermina Jasso

Department of Sociology

New York University

295 Lafayette Street, 4th Floor

New York, NY 10012-9605

USA

E-mail: gj1@nyu.edu

\footnotetext{
* I am grateful to Wil Arts, Gabrielle Ferrales, John Hagan, Samuel Kotz, Jui-Chung Allen Li, Stefan Liebig, Eva Meyersson Milgrom, Kjell Törnblom, Riel Vermunt, Murray Webster, and Bernd Wegener for many valuable discussions. This paper is forthcoming in: Kjell Törnblom and Riel Vermunt (eds.), Distributive and Procedural Justice: Research and Social Applications. London, UK: Ashgate.
} 


\section{INTRODUCTION}

In the study of distributive justice, there are two fundamental actors and three fundamental quantities. The observer reflects on the situation of a rewardee. The observer perceives the rewardee's actual reward, forms or retrieves an idea of the just reward for the rewardee, and assesses the fairness or unfairness of the actual reward, producing the justice evaluation. These three quantities form the crucial nexus. Though much comes before (rewardrelevant characteristics, for example) and much will follow (a strategy to alter the actual reward, for example), this trio occupies a central place, illuminating the essence of distributive justice processes and generating their long reach. ${ }^{1}$

The observer and rewardee may be the same person - in which case the situation is called reflexive - or they may be different persons - in which case the situation is called nonreflexive. Situations and research designs pertaining to justice for self thus involve reflexive quantities, those pertaining to justice for others involve nonreflexive quantities, and those pertaining to justice for all involve both reflexive and nonreflexive terms.

The reward may be any personal quantitative characteristic salient for individuals and societies, including goods (things of which more is preferred to less) and bads (things of which less is preferred to more). The quintessential good in the study of justice is money, in all its many forms, and the quintessential bad is time in prison. The one confers the illusion of perfection, as Weber (1904-1905/1958) understood, and the other destroys freedom - and perfection and freedom are among the fundamental engines of behavior (Jasso 2003a). Among children, an important good is the grades they receive in school, the natural counterpart to the income for which their parents work.

The actual reward is sometimes called the perceived-actual reward, because the observer's information about the rewardee's actual reward may be imperfect. Even in the

1 Sometimes there is a third actor - the allocator. In general, the allocator need not be a human person; it may be society or a deity. If human, the allocator need not be alive, as in the case of bequests and inheritance. 
reflexive case, if the reward under consideration is ordinal - such as beauty, intelligence, or athletic skill - so that measurement is by relative rank, the observer may misperceive his or her own relative rank.

Given that it is the perceived-actual reward which is compared to the just reward, leading to the assessment of justice or injustice, the perceived-actual reward plays an important part, in the spirit of Thomas Theorem, formulated by W. I. Thomas, which highlights the actor's definition of the situation.

Accordingly, in order to understand the justice evaluation - that is, to understand the observer's assessments of justice and injustice -- it is crucial to understand the observer's ideas of the rewardee's actual reward and just reward, termed, respectively, the observer-specific/ rewardee-specific perceived-actual reward and the observer-specific/rewardee-specific just reward. Hereafter, the phrases actual reward and just reward are shorthand for the longer expressions, and denoted $A$ and $C$, respectively; the justice evaluation is denoted $J$.

The three fundamental quantities $-A, C$, and $J$-may each be arrayed in an observer-byrewardee matrix, as depicted in Table 1. If observers and rewardees are the same, the matrix is square, and the principal diagonal contains the reflexive terms.

$$
\text { - Table } 1 \text { about here - }
$$

It is obvious that the justice evaluation is the outcome of a process in which the observer compares the actual reward to the just reward - a process captured in the justice evaluation function: ${ }^{2}$

$$
J=\theta \ln \left(\frac{A}{C}\right)
$$

where $\theta$ denotes the signature constant, to be described below. The actual reward and the just reward, too, are understood as the outcomes of two processes, called the actual reward function

2 Here we present the logarithmic-ratio specification of the justice evaluation function. For development of the general justice evaluation function and discussion of other potential specific forms of the justice evaluation functions, see Jasso $(1990,1999)$. 
and just reward function, respectively, and written:

$$
A=A(\boldsymbol{X}, \boldsymbol{Y} ; \varepsilon)
$$

and

$$
C=C(\boldsymbol{X}, \boldsymbol{Q} ; \varepsilon),
$$

where $\boldsymbol{X}, \boldsymbol{Y}$, and $\boldsymbol{Q}$ denote vectors of rewardee and situational characteristics -- the $\boldsymbol{X}$ vector affecting both the actual reward and the just reward -- and $\varepsilon$ and $\varepsilon$ denote stochastic errors.

The actual reward function (ARF), just reward function (JRF), and justice evaluation function (JEF) depicted in equations (1), (2), and (3) are three of the four fundamental functions in justice analysis (the fourth fundamental function being the justice consequences function, whose principal independent variable is $J$ ).

Estimation of the actual reward function and the just reward function yields the micro and macro effects known, in the $A$ case, as the effects of the reward-relevant characteristics and the reward inequality, and in the $C$ case as the principles of microjustice and macrojustice.

Note that the $A$ and $C$ functions can be jointly estimated, leading to assessment of the correlation among the unobservables in the two equations.

This paper develops several research designs for measuring and estimating the trio of fundamental justice quantities and the associated fundamental justice functions. Section 2 presents designs for measuring the actual reward and the just reward, both for self and others, including fictitious others. The protocols for measuring the actual reward and the just reward for fictitious others rely on the factorial survey method pioneered by Peter H. Rossi $(1951,1979)$. All the procedures for measuring the just reward discussed in Section 2 ask respondents directly what they think is the just reward, and thus have come to be called direct methods for approximating the just reward.

Section 3 begins by observing that the just reward obtained via the direct designs introduced in Section 2 may yield distorted measures of the just reward. Respondents may be reluctant to disclose their inner ideas of fairness or may feel the pressures of socialization and social desirability. This possibility, formalized by Jasso and Wegener (1997), led to the 
distinction between the true just reward and the disclosed just reward and to new efforts to devise techniques for estimating the true just reward.

Two keys make it possible to estimate the true just reward - the justice evaluation function and the factorial survey method. Section 3 summarizes the justice evaluation function.

With these two keys in place, we examine in Section 4 three indirect methods for estimating the true just reward, one pertaining to self, proposed by Evans (1989), and two pertaining to fictitious others and utilizing factorial survey methods, the first proposed by Jasso and Rossi (1977) and Jasso (1990) and the other proposed by Jasso and Webster (1999). As will be seen, the justice evaluation function and the factorial survey method are partners in a marriage of true minds. Indeed, in the case of one of the indirect methods, the progeny - estimates of the true just reward - depend equally on both parents, and cannot be obtained from one without the other. This indirect method is illustrated in Section 5.

\section{DIRECT MEASURES OF THE ACTUAL REWARD AND THE JUST REWARD}

We may conceptualize the actual reward and the just reward as corresponding to a person or to a particular characteristic. Because a person is a bundle of characteristics, we may think of the one-characteristic case as unidimensional and the person case as multidimensional. In the unidimensional case, the focal characteristic may be sex, age, schooling, occupation, and so on. For example, Leviticus (27:3-4) prescribes the value of a male (age 20-60) at fifty shekels and of a female (also 20-60) at thirty shekels; in this case, the dimension of interest is sex, and age is held constant for both sexes. The most widely studied characteristic in the unidimensional case is occupation. In the multidimensional case, characteristics typically include age, sex, education, occupation, and experience.

\subsection{Unidimensional Case - Occupation}

Four large survey projects have measured the actual reward and/or the just reward for particular occupations. 


\subsubsection{International Social Science Programme}

The International Social Science Programme (ISSP) has asked respondents, via the Inequality Modules fielded in 1987, 1992, and 1999, to provide both the actual reward and the just reward for sets of 9-11 occupations. Table 2 reports the occupational titles in each of the four surveys. ${ }^{3}$

\section{- Table 2 about here -}

The wording of the two questionnaire items is as follows:

Actual Reward Questionnaire Item. "We would like to know what you think people in these jobs actually earn. Please write in how much you think they usually earn, each year, before taxes. Many people are not exactly sure about this but your best guess will be close enough. This may be difficult, but it is very important. So please try. Please write in how much they actually earn each year.”

Just Reward Questionnaire Item: "Next, what do you think people in these jobs ought to be paid -- how much do you think they should earn each year before taxes, regardless of what they actually get? Please write in how much they should earn each year."

\subsubsection{International Social Justice Project}

The International Social Justice Project (ISJP) has asked respondents in its fieldings of 1991, 1996, 2000, and 2006 to provide the actual reward and the just reward for two of the occupations studied in the ISSP. The two occupations are "unskilled worker in a factory" and "chairman of a large national corporation" (Table 2$){ }^{4}$

The wording of the two items is as follows:

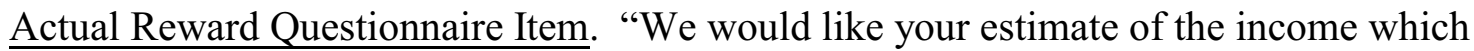
people in some occupations actually earn per (YEAR/MONTH) on average. (Your best guess

3 For further information on the ISSP program of surveys, documentation, and public-use data, see www.issp.org .

4 For further information on the ISJP program of surveys, documentation, and public-use data, see www.butler.edu/isjp/. 
will be fine.)"

Just Reward Questionnaire Item. "You have told me what you think people with these occupations actually earn. Now tell me what you think would be a just and fair average (YEARLY/MONTHLY) income for people in these occupations.”

\subsubsection{New Immigrant Survey}

The U.S. New Immigrant Survey (NIS), in the first round of surveying the 2003 immigrant cohort (NIS-2003-1), asked respondents to provide the actual reward for seven occupations, both in the United States and in their country of last residence. The seven occupations consisted of the two studied in both the ISSP and the ISJP - unskilled worker in a factory and chairman of a large national corporation - plus five other occupations randomly selected from among 10 occupations, 7 of which were studied in the ISSP (Table 2). ${ }^{5}$

The wording of the two items is as follows:

Actual Reward Questionnaire Item - United States. "We would now like to know what you think people in various jobs actually earn in the United States. Please state how much you think workers usually earn, each year, before taxes. Many people are not exactly sure about this, but your best guess will be close enough. This may be difficult, but it is very important, so please try. Please estimate in US dollars how much each worker actually earns per year before taxes."

Actual Reward Questionnaire Item - Country of Last Residence. "We are also interested in what you think workers in these jobs earned in your country of last residence just before you came to the United States. Please make your estimates in the currency of that country. How much do you think each worker earned per year in your country of last residence before taxes?"

\subsubsection{German Socio-Economic Panel}

The German Socio-Economic Panel (GSOEP) asked respondents in its fielding of 2005 to provide the actual reward and the just reward for versions of the two occupations studied in the

5 For further information on the NIS, documentation, and public-use data, see http://nis.princeton.edu . 
ISSP and the ISJP. The two occupations are "unskilled worker" and "manager on the board of directors of a large company" (Table 2). ${ }^{6}$

The wording of the two items is as follows:

Actual Reward Questionnaire Item. "How high on average is the monthly net income of an [ ]?"

Just Reward Questionnaire Items. The actual reward item is followed by two items. First, the respondent is asked, "Would you say that this income has a just relation to the job demands?" If the response is, "No, the respondent is asked the further question, "How high would a just monthly income of [__ $]$ be, from your point of view?"

\subsection{Multidimensional Case - Self and Other}

A person is a combination of many characteristics. It is a person who receives an actual reward, and it is a person about whom many judgments of just rewards are made. When the rewardee is self - i.e., in the reflexive case - the multidimensionality is implicit. Similarly, when the rewardee is a named other, the multidimensionality, too, may be implicit. Yet there is substantial interest in assessing ideas of the worth of characteristics and in moving from the particular to the abstract. To estimate the effects of rewardee sex, age, schooling, experience, etc., on observers' ideas of the just reward, a sharp instrument is the factorial survey, in which fictitious persons are generated by randomly combining many levels of many characteristics. In all these situations, the actual and just rewards can be directly measured.

\subsubsection{Actual and Just Reward for Self}

Actual Reward for Self. In the domain of earnings, the actual reward for self is measured in virtually all surveys and censuses. These include, besides the four data sets in Table 2, national censuses, the major cross-sectional data bases (such as the General Social Surveys), and the major longitudinal data bases (such as the National Longitudinal Studies of Labor Market

6 For further information on the GSOEP, documentation, and public-use data, see http://www.diw.de/english/sop/index.html . 
Experience, the Panel Study of Income Dynamics, and the Health and Retirement Survey).

For example, actual earnings was measured in the ISJP-1991 via the question: "What is the income you yourself received from a job or business (BEFORE/AFTER) taxes) (during 1990/in last 12 months/per month)?” In the New Immigrant Survey, a large set of questions tap wages, salaries, overtime pay, and so on, for each job a respondent has.

Surveys on students also routinely ask for information about the student's grades. For example, the Israeli Junior High School Study (IJHSS), fielded in 1986, asked students to report their grades in five subjects (Dar and Resh 1993, 1996). The questionnaire item asked: "What was the grade you received in [subject] in the last semester?"

Once the actual rewards for self are measured, it is straightforward to estimate the actual reward function for a population - for example, wage attainment and grade attainment functions. These are among the most intensely studied relations in all social science.

$\underline{\text { Just Reward for Self. }}$ The just reward for self is a more specialized quantity, and it is not routinely measured. The IJHSS and the ISJP innovated with inclusion of direct measures of the just reward. The questionnaire item in the IJHSS states: "What is the grade you think you deserved to get in [subject]?" The questionnaire item in the ISJP states: "What income do you feel you deserved from your (job/business)?" The GSOEP (2005) asks two questions, "Is the income that you earn at your current job just, from your point of view?" and if the answer is negative, follows with, "How high would your net income have to be in order to be just?"

As with the actual rewards, once the just rewards for self are obtained, it is straightforward to estimate the just reward function for a population. Examples include Jasso and Wegener (1999) and Jasso and Resh (2002).

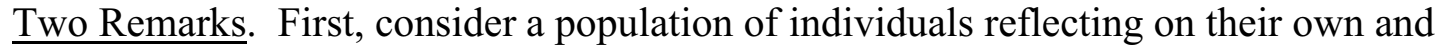
others' rewards. Measurement of the actual and just rewards for self generates the entries for the principal diagonal of the $\boldsymbol{A}$ and $\boldsymbol{C}$ matrices in Table 1. Second, note that whenever both the actual and just rewards for self are obtained in the same survey (as they were in both the IJHSS and the ISJP), it becomes possible to estimate jointly the actual reward equation and the just 
reward equation, thus obtaining a window into the correlation between the unobservables in the two equations.

\subsubsection{Actual and Just Reward for Fictitious Others}

The factorial survey method pioneered by Peter H. Rossi and developed with associates (Rossi et al. 1974, Rossi 1979, Rossi and Anderson 1982, Rossi and Berk 1985, Jasso 2006b) provides a systematic way to generate fictitious individuals whose actual and just rewards can be directly measured. Briefly, the investigator (1) chooses a set of reward-relevant characteristics (such as age, sex, schooling, experience); (2) chooses a set of levels for each characteristic (e.g., two sexes, 10 levels of age, 12 levels of schooling, etc.); (3) randomly combines all levels of all characteristics to obtain the population of fully crossed characteristics; and (4) deletes impossible combinations (such as a 25-year-old with 30 years of work experience). This adjusted population of fictitious individuals, which can be very large, is then the sampling frame from which random samples are drawn for presentation to respondents. The size of the random samples is roughly in the 30-60 range, enabling assessment of respondent-specific equations of the effects of rewardee characteristics on the actual reward and the just reward. Each description of a fictitious person is called a vignette; thus, each respondent judges a deck of vignettes. ${ }^{7}$

Direct Measure of the Actual Reward for Fictitious Other. If the research objective is to obtain respondent perceptions of the actual reward, then the task assigned to the respondent is to provide an estimate of the actual reward for each vignette. For an example of this design, we turn to the ongoing research on executive compensation carried out by Jasso and Meyersson (unpubl). In this research, fictitious chief executive officers (CEOs) were generated by randomly combining the levels of such characteristics as age, sex, industry sector, and firm capitalization. Table 3 presents the characteristics and their levels. Table 4 provides a facsimile of the instructions to respondents. Table 5 depicts a sample vignette with space for the respondent to write in the perceived actual reward.

7 A brief introduction to Rossi's factorial survey method is provided in Jasso (2003b). 
- Tables 3, 4, and 5 about here -

The obtained data make it possible to estimate for each respondent his or her own actual reward function, and hence to assess interrespondent variability in perceptions of actual returns to reward-relevant characteristics. This type of equation is an example of the positive-beliefs equation described and analyzed in Jasso (2006b).

Direct Measure of the Just Reward for Fictitious Other. If the research objective is to obtain respondent perceptions of the just reward, the task involves providing direct estimates of the just reward. For an example of this design, we consider the ongoing research on prison sentences carried out by Hagan, Ferrales, and Jasso (unpubl). In this research, judicial case scenarios consisting of fictitious prison guards accused of torturing fictitious prisoners were generated by randomly combining the levels of pertinent case characteristics, including characteristics of both prison guards and prisoners. Respondents are then asked to provide their idea of the just prison sentence for the accused prison guards.

Data obtained in this design enable estimation of each respondent's just reward equation, and hence assessment of interrespondent variability in the principles of microjustice. In this case, the equation is of the normative-judgments type described and analyzed in Jasso (2006b).

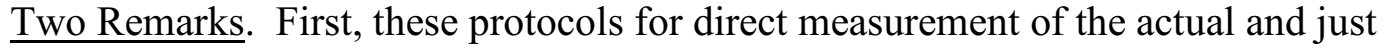
rewards in factorial survey studies yield estimates for nonreflexive $\boldsymbol{A}$ and $\boldsymbol{C}$ matrices. The observers and rewardees are two different sets of persons - the one a set of real persons, the other a set of fictitious persons - and the matrices need not be square. Importantly, every cell is susceptible of being populated (if every respondent provides an estimate for every vignette). Second, if the research design calls for all respondents to provide both actual rewards and just rewards, then the actual reward equation and the just reward equation can be estimated jointly and, as in the case of justice for self described above, the correlation between the unobservables can be explored. 


\section{INTERLUDE: DIRECT AND INDIRECT MEASURES OF THE JUST REWARD}

Our purpose is to approximate the actual reward and the just reward and to estimate the actual reward function and the just reward function. It would seem that we have achieved our purpose. This article could come to a close right here, or at least the expository part before providing an illustration. We have laid out methods for every case of interest -- the actual reward and the just reward for both self and other. We could pack up and go home.

There is a nagging thought, however, one that never fails to surface in reviewers' comments and in colloquia, and it affects the just reward. How do we know that the estimates provided by respondents represent their true ideas of justice? The survey research enterprise has long been cognizant of response effects and social desirability bias. Suppose that there is a discrepancy between what respondents say and what they really think. Suppose that there are two just rewards rather than one - the true just reward and the disclosed just reward.

Such were the concerns that led Jasso and Wegener (1997) to propose the existence of two just rewards and to posit a disclosure function which converts the true just reward into the disclosed just reward. It was already thought that both the just reward and the justice evaluation exhibit variation across different contexts. These contexts are formalized by the mnemonic "brots" - where each letter stands for a context which may differentially shape the operation of the sense of justice: $b$ for the benefit or burden under consideration, $r$ for the type or identity of the rewardee, $o$ for the observer, $t$ for the time period, and $s$ for the society.

Now Jasso and Wegener (1997) argued that the discrepancy between the true just reward and the disclosed just reward may differ systematically not only by the five brots dimensions but by more specific elements within the brots dimension, such as observer age and gender. And they suggested that research was needed to document this discrepancy and its correlates. Such work would yield a protocol for calibration as well as a clear sense of the kinds of respondents or situations not susceptible of disclosure mechanisms and thus on whom direct methods can be safely used versus the kinds of respondents and situations for which indirect methods are 
superior.

But first, it was necessary to examine the possibilities for indirect measurement of the just reward. How could it be done? Perhaps projective techniques? Perhaps designs utilizing advances in brain imaging. How could one get inside a person's head and capture the true just reward?

Help would come from an unexpected quarter - the justice evaluation function. Indeed, two indirect procedures for estimating the just reward had already been proposed, one for self by Evans (1989) and one for fictitious others by Jasso (1990), the latter using data collected by the method developed in Jasso and Rossi (1977).

To understand these and a third indirect method, we begin with an overview of the justice evaluation function.

Justice Evaluation Function. As discussed above, the observer compares the rewardee's actual reward to the observer's idea of the just reward for the rewardee, producing the experienced justice evaluation, denoted $J^{*}$ :

$$
J^{*}=\ln \left(\frac{A}{C}\right) .
$$

If this mathematical relation faithfully represents the justice evaluation process, then it could be used to construct an indirect measure of the just reward. For the fundamental comparison at the heart of a justice evaluation is between the actual reward and the true just reward, and thus if we know the actual reward and the justice evaluation, we can solve for the just reward!

But we were not out of the woods yet. Two challenges remained. First, it was important to think hard about the correct form of the justice evaluation function, continuing the work reported in Jasso (1990). Second, the formula in (4) represents the experienced justice evaluation function, and work by Jasso (1980) and Jasso and Wegener (1997) indicated that the justice evaluation, too, was susceptible of distortion in the empirical context, and could be thought of as two justice evaluations, the experienced justice evaluation and the expressed justice evaluation. 
The Form of the Justice Evaluation Function. The log-ratio form in (1) and (4) possesses several useful and appealing properties. The first three noticed were: (1) exact mapping from combinations of $A$ and $C$ to $J$; (2) integration of rival conceptions of $J$ as a ratio (Homans 1974 , 1976) and as a difference (Berger et al. 1972); and (3) deficiency aversion, viz., deficiency is felt more keenly than comparable excess (and loss aversion, viz., losses are felt more keenly than gains). These properties were quickly discussed (e.g., Wagner and Berger 1985) and remain the most often cited (Whitmeyer 2004). In the course of further scrutiny of the JEF, two new properties emerged: (4) additivity, such that the effect of $A$ on $J$ is independent of the level of $C$, and conversely; and (5) scale invariance (Jasso 1990). Six years later two other desirable properties were noticed: (6) symmetry, such that interchanging $A$ and $C$ changes only the sign of $J$; and (7) the fact that the log-ratio form of the JEF is the limiting form of the difference between two power functions,

$$
\lim _{k \rightarrow 0} \frac{A^{k}-C^{k}}{k}=\ln \left(\frac{A}{C}\right)
$$

which both strengthens integration of the ratio and difference views and also integrates powerfunction and logarithmic approaches (Jasso 1996). More recently, an eighth (almost magical) property has come to light, linking the JEF and the Golden Number, $(\sqrt{5}-1) / 2$ (Jasso unpubl).

The logarithmic-ratio form is the only functional form which satisfies both scale invariance and additivity (Jasso 1990).

The JEF connects the two great literatures in the study of justice, the literature on ideas of justice and the literature on reactions to injustice. As well, it generates several useful links, via the justice index (the arithmetic mean of $J$ ): (1) a link between justice and two measures of inequality, Atkinson's $(1970,1975)$ measure defined as one minus the ratio of the geometric mean to the arithmetic mean and Theil's MLD; (2) a link with ideology, via decomposition of the justice index into the amount of overall injustice due to reality and the amount due to ideology; and (3) a link with poverty and inequality, via another decomposition of the justice index into the amount of overall injustice due to poverty and the amount due to inequality (Jasso 1999). 
Of course, scientific questions are never completely closed. To that end, it is useful to examine alternative functional forms, to consider which features of the log-ratio form are too important to give up (say, loss aversion and scale invariance?), and to empirically try alternate forms. Work with alternative forms is reported in Jasso (1990, 1996, 2006b), the last article reporting an empirical analysis using the power-difference form in (5).

Note that the indirect method for measuring the just reward to be presented below can be used with any additive functional form, as illustrated by the analysis in Jasso (2006b).

Experienced and Expressed Justice Evaluations. The experienced justice evaluation $J^{*}$ is the quantity that generates behavioral consequences. However, individuals differ in their style of expression, some given to hyperbole, others to understatement, and thus the experienced justice evaluation is transformed into the expressed justice evaluation $J$ (Jasso 1980, Jasso and Wegener 1997). Meanwhile, justice evaluations arise about both goods and bads. Accordingly, justice analysis introduces a quantity called the signature constant and denoted $\theta$; by its sign the signature constant indicates whether the observer regards the reward as a good or as a bad, and by its absolute value the signature constant indicates expressiveness. Thus, the justice evaluation function becomes, as shown in (1) and repeated here:

$$
J=\theta \ln \left(\frac{A}{C}\right) .
$$

The True Just Reward. Immediately, a new expression for the true just reward is generated:

$$
C=A \exp (-J / \theta) .
$$

This new expression points the way to systematic construction of indirect methods for estimating the true just reward. Look at expression (7). The true just reward is a function of three quantities: the actual reward, the expressed justice evaluation, and the signature constant. Accordingly, the true just reward can be estimated from a design in which (1) the investigator provides the actual reward, (2) the respondent provides the expressed justice evaluation, and (3) the signature constant is estimated. 
A New Approach. The road is becoming clear. To transform the theoretical (mathematical) justice evaluation function into an empirical (statistical) justice evaluation function, we may write, for every judgment made by every respondent:

$$
\text { expressed justice evaluation }=\theta \ln (\text { actual reward })-\theta \ln (\text { just reward })+\varepsilon \text {. }
$$

The just reward is unobserved, of course. If a respondent makes several judgments, these can be aggregated according to the usual regression setup, producing the estimable form:

$$
\text { expressed justice evaluation }=\alpha+\theta \ln (\text { actual reward })+\varepsilon .
$$

The exact interpretation of equation (9) and, in particular, the properties of theta depend on the research design. A priori, we can distinguish two possible research designs. In the first research design, the respondent judges the fairness or unfairness of several actual rewards for a single rewardee. This case, now called the multiple-rewards-per-rewardee design, generates a constant just reward, the constant is absorbed into the equation intercept, and estimation of $\theta$ is straightforward. This design was developed by Evans (1989) for estimating the just reward for self and by Jasso and Webster (1999) for estimating the just reward for fictitious others.

In the second research design, the respondent judges the fairness or unfairness of one actual reward for each of several rewardees. This case, now called the one-reward-perrewardee design, generates as many just rewards as there are rewardees. Equation (9) is straightforwardly estimated, but because there are many unobserved just rewards - one for each actual reward -- it is urgent to guard against correlation between the actual rewards and the unobserved just rewards. Any such correlation would bias the estimate of the signature constant, as discussed below.

Properties of Theta. The signature constant theta does a lot of work. It enables estimation of both the experienced justice evaluation and the true just reward. Thus, the utmost care must be taken in estimation of theta. Jasso (1990) pointed out that if the actual rewards are uncorrelated with the unobserved just rewards, then the estimate of theta is unbiased and consistent and, under the assumption that the errors are independently distributed with mean zero and constant variance, is best linear unbiased (BLUE). However, if the actual rewards are 
correlated with the unobserved just rewards, then the estimate of theta obtained from equation (9) is biased. Thus, the challenge in the one-reward-per-rewardee design is to satisfy orthogonality. And, indeed, it is this danger of failure to satisfy orthogonality that led Jasso and Webster (1999:370-372) to develop the multiple-rewards-per-rewardee design.

Orthogonality and the Factorial Survey Method. The solution to the problem of estimating theta in the one-reward-per-rewardee design lay in the researchers' backyard. Jasso had begun her justice work with a vignette study, under the tutelage of Peter H. Rossi, who pioneered the factorial survey method. Look again at equations (8) and (9). In the factorial survey designs presented in Section 2, fictitious individuals are generated by randomly combining all the levels of a set of potentially reward-relevant characteristics. If the design is modified to include an actual reward randomly attached to each fictitious individual, then the orthogonality condition is exactly met - there is zero correlation between the actual reward and the reward-relevant characteristics and, hence, the unobserved just reward. It follows that the estimate of theta is unbiased and consistent and indeed, as already noted, may be BLUE.

Further methodological issues remain to be discussed, including properties of the estimated just rewards and such additional features as emphasizing in the instructions to respondents that the actual rewards are random, so that respondents will not infer a correlation between actual reward and just reward. Section 4 covers these matters.

But for now we can celebrate a wedding. In the one-reward-per-rewardee research design, the marriage of the justice evaluation function and the factorial survey method enables unbiased and consistent estimation of the signature constant theta, and the signature constant theta in turn enables estimation of the experienced justice evaluation and the true just reward.

\section{TWO INDIRECT METHODS FOR ESTIMATING THE JUST REWARD: THE ONE-REWARD-PER-REWARDEE METHOD AND THE MULTIPLE-REWARDS-PER-REWARDEE METHOD}

This section describes two indirect methods for estimating the just reward for fictitious 
others, an early love child and a late love child, as it were, of the marriage of the justice evaluation function and the factorial survey method, and describes as well an indirect method for estimating the just reward for self, a middle child not requiring the factorial survey method. The data collection protocol for the early love child follows exactly and in every particular the design formulated by Jasso and Rossi (1977), an application to the study of justice of the factorial survey method pioneered by Rossi. The data collection protocol for the late love child, developed by Jasso and Webster (1999), is a modification of the initial protocol. The data analysis protocols for both methods developed over the years, as experience with the factorial survey method and the justice evaluation function revealed new possibilities. The indirect method for estimating the just reward for self, developed by Evans (1989) and first implemented

in the 1987 round of the International Social Science Survey / Australia (Kelley and Evans 1987), is discussed together with the Jasso and Webster (1999) design, as they are both multiplerewards-per-rewardee methods.

As in all factorial survey studies, the data collection protocol in the two designs for fictitious others has three elements, a respondent sample - omitted from consideration here - a vignette sample, and a rating task. General topic-independent procedures for both data collection and data analysis in factorial surveys are described in Jasso (2006b). In the data analysis summaries below, we focus on estimation of the true just reward.

\subsection{The One-Reward-Per-Rewardee Method}

In the one-reward-per-rewardee method, each respondent is given descriptions of a set of rewardees and asked to judge the fairness of the (one) actual reward attached to each rewardee. This is the original design developed by Jasso and Rossi (1977).

\subsubsection{Data Collection Protocol}

Vignette Sample. The design calls for generating the population of all possible combinations of all levels of a set of variables including (a) potentially reward-relevant characteristics and (b) the actual reward. This population is then adjusted by deleting any impossible combinations (such as a physician who only completed the eighth grade). Random 
samples are then drawn from the adjusted population. Note that the zero correlation between the actual reward and each of the reward-relevant characteristics is unimpaired by deletion of the impossible combinations. Thus, the data satisfy the orthogonality condition discussed above.

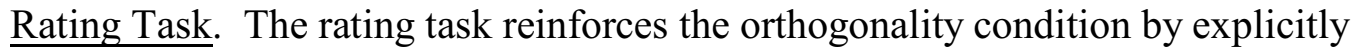
describing the actual reward as "hypothetical" and "randomly attached" to the fictitious individual. Table 6 presents a facsimile of the instructions to respondent in the one-reward-perrewardee method.

- Table 6 about here -

Table 7 provides an example of the vignettes in this design.

- Table 7 about here -

\subsubsection{Data Analysis Protocol}

Recall the basic justice evaluation regression equations in (8) and (9). According to justice theory, the respondent forms or retrieves a true just reward using the information in the reward-relevant characteristics, next compares the actual reward to the true just reward, producing an experienced justice evaluation which is transformed by the signature constant theta into the numerical rating $J$. Thus, the "silent partner" in the ratings is the set of unobserved true just rewards.

The justice evaluation regression equation in (8) may be re-written to express the estimated true just reward as a function of the actual reward $A$, the estimated signature constant theta, and the expressed justice evaluation $J$ :

$$
\text { estimated true just reward }=\text { actual reward } \times \exp \left(-\frac{\text { expressed justice evaluation }}{\text { estimated } \theta}\right)
$$

or, more compactly:

$$
\hat{C}=A \exp (-J / \hat{\theta}) .
$$

Estimation of equation (9), separately for each respondent, yields each respondent's estimated theta. As already noted, this estimate is unbiased and consistent and may be BLUE.

The estimated true just rewards, being nonlinear transformations of estimated theta, lose 
the property of unbiasedness but by Slutsky's theorem retain the property of consistency. This is one of the reasons why premium is placed on having each respondent judge a large number of vignettes.

The estimated true just rewards are ready to be displayed in the just reward matrix (as in Table 1, panel 2) and used in analyses of the just reward function and the just reward distribution to obtain the estimates of the principles of microjustice and the principles of macrojustice.

Section 5 provides an illustration of the one-reward-per-rewardee indirect method for estimating the just reward.

\subsection{The Multiple-Rewards-Per-Rewardee Method}

Evans (1989) developed a procedure for measuring just earnings for self, in which each respondent was given five hypothetical earnings amounts for self and asked to judge the fairness or unfairness of each. This procedure was first applied in the 1987 round of the International Social Science Survey / Australia (Kelley and Evans 1987). Jasso and Webster (1999) developed a procedure for measuring the just reward for others, in which each respondent was given seven hypothetical earnings amounts for each of ten fictitious rewardees and asked to judge the fairness or unfairness of each.

Both the Evans (1989) for-self procedure and the Jasso and Webster (1999) for-others procedure share the same basic data analysis protocol. Data collection in the for-self procedure is straightforward; the investigator presents a set of hypothetical actual rewards, and asks the respondent to judge the justice of each. Here we describe the data collection protocol for the forothers procedure, which utilizes factorial survey methods. This method was designed to overcome the danger that respondents, despite the labeling of the actual reward as random, might inject a correlation between the actual rewards and the reward-relevant characteristics, thus damaging the good properties of the estimated theta.

\subsubsection{Data Collection Protocol in the For-Others Application} of the Multiple-Rewards-Per-Rewardee Method

Vignette Sample. In the for-others application of the multiple-rewards-per-rewardee 
method, the design begins with the population of all possible combinations of all levels of a set of potentially reward-relevant characteristics. This population is next adjusted by deleting any impossible combinations (such as a physician who only completed the eighth grade). Random samples are then drawn from the adjusted population. Finally, a set of rewards randomly drawn from a larger set of rewards is attached to each fictitious individual. In this design, not only is there zero correlation between the actual rewards and the reward-relevant characteristics, but the presence of several hypothetical actual rewards underscores their random nature and averts the possibility of the respondent seeing a link between reward and reward-relevant characteristic. This design a fortiori satisfies the orthogonality condition.

$\underline{\text { Rating Task. }}$ The rating task requests that the respondent judge the fairness or unfairness of each of the actual rewards attached to each rewardee. Table 8 presents a facsimile of the instructions to respondent in the multiple-rewards-per-rewardee method.

- Table 8 about here --

Table 9 provides an example of the vignettes in this design.

- Table 9 about here --

\subsubsection{Data Analysis Protocol}

As before, recall the basic justice evaluation regression equations in (8) and (9). When the respondent judges the fairness of several potential actual rewards for a particular rewardee (self or fictitious other), the just reward (in the respondent's mind) is constant and can be retrieved via estimation (separately for each respondent-rewardee combination) of the regression equation (9). It will be useful to have distinctive notation for the multiple-rewards-per-rewardee method, and thus we re-write equations (8) and (9) for this case:

$$
\begin{aligned}
J & =\theta \ln A-\theta \ln C+\varepsilon \\
& =\theta \ln A+v+\varepsilon .
\end{aligned}
$$

Under the assumption that the errors are independently distributed with zero mean and constant variance across the multiple ratings within the respondent-rewardee combination, the slope $\theta$ in the second equation in (12) provides an unbiased and consistent estimate of the signature 
constant, and the intercept $v$ provides an unbiased and consistent estimate of the negative of the product of the signature constant $\theta$ and the log of the just reward. Thus, the estimate of the respondent-specific/rewardee-specific true just reward is obtained by the following formula:

$$
\hat{C}=\exp (-\hat{\mathbf{v}} / \hat{\theta})
$$

Because the estimated just rewards are nonlinear transformations of the slope and intercept in (12), they lose the unbiasedness property; however, by Slutsky's theorem they retain consistency. $^{8}$

For illustrations of the for-others application of the multiple-rewards-per-rewardee method, see Jasso and Webster (1999) and Jasso (2006a). The latter analysis shows how this design enables test of two new kinds of impartiality, framing-impartiality and expressivenessimpartiality.

\section{ILLUSTRATION: MEASUREMENT AND ESTIMATION IN A FACTORIAL SURVEY OF THE JUSTICE OF EARNINGS USING THE ONE-REWARD-PER-REWARDEE METHOD}

To illustrate, we use the data for one respondent from a larger study described in Jasso (unpubl). Like the other respondents, this respondent judged the justice of the earnings of 20 fictitious workers, providing for each one an expressed justice evaluation. Each worker was described in terms of earnings-relevant characteristics. The descriptions were generated according to the standard Rossi factorial survey protocol, in which all the levels of all characteristics are fully crossed, with impossible combinations (such as a physician with an eighth-grade education) deleted. A hypothetical earnings amount was randomly attached to each description. Tables 10 and 11 present the instructions for the rating task and an example of a

8 This design enables a new possibility for the signature constant, namely, that not only does it differ across respondents but also that, within respondent, it differs across rewardee. The procedure described in the text and equation (13) pertain to the case in which theta is rewardeespecific. If statistical tests indicate that theta does not differ across rewardees, then the procedure for estimating the true just reward differs from the procedure in the text. 
vignette, respectively. Note that the rating task uses the number matching technique pioneered by Stevens (1975) in which each respondent is given maximal freedom to map the subjective justice continuum onto numbers. Note also that the real-number continuum is activated by explicitly mentioning decimals and fractions in the instructions.

- Tables 10 and 11 about here -

Table 12 presents, in the two leftmost columns of panel $\mathrm{A}$, the raw data for one respondent, namely, the actual earnings randomly attached to the fictitious worker and the respondent's expressed justice evaluation. Estimation of equation (9), that is, regression of the expressed justice evaluation on the natural log of actual earnings, yields an estimate of the signature constant $\theta$ of 5.77 (panel B). ${ }^{9}$

- Table 12 about here --

As evident in equations (4) and (6), the experienced justice evaluation for a particular worker, denoted $j_{r}^{*}$, is estimated by dividing the expressed justice evaluation, denoted $j_{r}$, by the expressiveness coefficient:

$$
\hat{j}_{r}^{*}=\frac{j_{r}}{|\hat{\theta}|}
$$

The estimated experienced justice evaluations, corresponding to each of the fictitious workers, are reported in the third column of panel A. For example, this respondent's expressed justice evaluation for Worker \# 1 was +2.5 (Table 12). The estimated signature constant was 5.77 . The estimated experienced justice evaluation is thus

$$
\hat{j}_{1}^{*}=\frac{j_{1}}{|\hat{\theta}|}=\frac{2.5}{5.77} \approx .433 .
$$

It is useful that the justice evaluation function, in combination with the factorial survey

9 The raw data in Table 12 can be used by the interested reader to generate everything else in Table 12 - the estimates of the justice evaluation function, the experienced justice evaluations, and the true just rewards - as well as all the estimates in Table 13 except those of the principles of microjustice. 
justice design, makes it possible to estimate the experienced justice evaluation from a single datum obtained from the respondent - the expressed justice evaluation. But what is truly remarkable is that this same marriage of the justice evaluation function and the factorial survey justice design also makes it possible to estimate the true just reward. Recall the expressed justice evaluation function in (6); there are four quantities, the expressed justice evaluation, the actual reward, the true just reward, and the signature constant. The factorial survey justice design provides the actual reward, the expressed justice evaluation is obtained from the respondent, and the signature constant is estimated via statistical estimation of the equation (as shown above). Thus, there remains only one unknown -- the true just reward.

Using formula (11) we obtain estimates of the true just reward in the eyes of each respondent for each fictitious worker. Continuing with the illustration referring to one respondent, Table12 reports, in the rightmost column of panel A, the estimated true just reward amounts. The estimated true just reward for Worker \# 1 is $\$ 16,211$, obtained by applying formula (11): ${ }^{10}$

$$
25000 \times \exp (-2.5 / 5.77) \approx 16211 .
$$

Now that the just rewards have been estimated, we can confirm that, indeed,

$$
\begin{aligned}
& \hat{j}_{1}^{*}=\ln \left(\frac{a_{1}}{\hat{c}_{1}}\right)=\ln \left(\frac{25,000}{16,211}\right) \approx .433 \\
& j_{1}=\hat{\theta} \ln \left(\frac{a_{1}}{\hat{c}_{1}}\right)=5.77 \times .433 \approx 2.5 .
\end{aligned}
$$

Of course, the estimated justice evaluation equation provides further information. First, the sign of the signature constant, known as the framing coefficient, indicates whether the respondent regards the reward as a good or a bad; in this case, the sign is positive, indicating that the respondent regards earnings as a good.

10 The just reward amounts in Table 12 are calculated using a less rounded version of the signature constant than 5.77. Thus, arithmetic computation using the rounded version shown in expression (17) yields an estimate of the true just reward slightly different from the one shown. 
Second, the intercept in the justice evaluation equation provides the product of $-\theta$ and the expected value of the logged just earnings amounts, and we can confirm that this quantity indeed equals approximately -57.4 .

Third, the equation $R^{2}$ indicates whether the actual inequality, put experimentally into the actual rewards, is regarded by the respondent as larger or smaller than the just inequality. If a respondent's justice evaluation function has an $R^{2}$ larger than .5 , the respondent considers the actual inequality to be higher than the just inequality, and if a respondent's justice evaluation function has an $R^{2}$ smaller than .5 , the respondent considers the actual inequality to be lower than the just inequality. ${ }^{11}$ In this case, the value of $R^{2}$ is .912 , which exceeds .5 , and thus this respondent considers the actual inequality to be larger than the just inequality.

Now that the experienced justice evaluations and the true just rewards have been estimated, it becomes possible to estimate the many quantities and relations for which the just reward and the justice evaluation function are the basic ingredients - the quantities and relations collected under the rubric of nonfundamental quantities and relations in Jasso and Wegener (1997:416), as augmented by the more recent work in Jasso and Webster $(1997,1999)$ and Jasso (1999) - and thus to strike out in a number of new research directions. First, the just reward function can be estimated, giving rise to the estimated principles of microjustice. Second, the principles of macrojustice can be estimated by calculating measures of location and inequality in the distribution of the just rewards. Third, just gender earnings gaps can be calculated. Fourth, each respondent can be characterized by the proportions of rewardees that he/she regards as underrewarded, justly rewarded, and overrewarded. Fifth, the experienced justice evaluations can be used to calculate the justice indexes, and thence decomposition into the amount of injustice due to poverty and the amount due to inequality.

For example, to estimate the respondent-specific just reward functions, we regress,

11 This interpretation is derived by analyzing the variance decomposition; fuller details are provided in Jasso (unpubl). 
separately for each respondent, the natural logarithm of the just reward on the rewardee characteristics. The obtained estimates, or transformations thereof, constitute estimates of the respondent-specific principles of microjustice. To illustrate, in a just earnings function, the coefficient of schooling provides an estimate of the just return to investment in an additional year of schooling. The exponential of the coefficient of the binary sex variable, measures the gender multiplier; women are coded " 1 " and thus the multiplier is applied to the earnings of females, so that subtracting one yields the tax (if negative) or bonus (if positive) on women's earnings, relative to the earnings of comparable men, in percentage points. The gender multiplier has a natural interpretation as the ratio of female to male earnings; a gender multiplier of .8 would indicate the view that the just earnings for a woman is $80 \%$ of the just earnings of a comparable man.

All of the foregoing estimation and analysis is carried out for each respondent separately. A further set of analyses compares the respondents. The distributions of each respondentspecific estimate - for example, the distributions of the principles of microjustice and macrojustice - are inspected to establish the extent of disagreement on each principle. A variety of statistical tests are carried out to assess respondent differences on expressiveness, inequality in the just reward distribution, and so on. Multilevel methods provide for the joint estimation of many of these quantities and equations, as discussed in Jasso (2006b).

Here we continue with our illustration focusing on a single respondent. Table 13 presents all the quantities estimated for this one respondent. For clarity, the quantities are grouped into two sets, those pertaining to the first central question in the study of justice and those pertaining to the third central question. As shown, the estimates of the just earnings function indicate that this respondent considers the just base wage for a male to be approximately $\$ 10,207$ (panel A.1). This respondent views the just rate of return to schooling as 5.6 percent for each additional year of schooling.

- Table 13 about here -

Table 13 also reports, in panel A.2, estimates of the principles of macrojustice guiding 
this respondent's ideas of the just earnings. Three measures of inequality are provided, together with the just relative minimum - the just minimum income is slightly more than half the mean and the relative maximum - a low 1.6. Not surprisingly, Plato's ratio (the ratio of the maximum to the minimum) is slightly over 3, smaller than most estimates of the actual value of Plato's ratio and smaller even than the 5 which for Plato signaled extreme inequality. As is well-known, different measures of inequality capture different features of a distribution, and thus a set of five indicators provides a fuller picture of the respondent, and will in particular be useful when this respondent is compared to the others in the study.

As well, Table 13 provides three different ways of assessing the respondent's views on gender and justice. The just gender multiplier, estimated from the just earnings function, and the mean-based gender gap both indicate that, in the eyes of this respondent, women should earn more than otherwise comparable men. However, the median-based gender gap indicates the opposite. As with inequality, the set of three measures provide a fuller picture of the respondent than a single measure would provide; and this will be especially useful when this respondent is compared with the other respondents.

This respondent also judged half the fictitious workers to be overpaid, $40 \%$ to be underpaid, and only ten percent to be justly paid (Table 13, panel B.1). Moreover, the justice index JI1 estimated for this respondent is positive, indicating that the center of gravity of his/her justice evaluations lies in the overreward region of the justice evaluation scale (Table 13, panel B.2). The decomposition of JI1 further indicates that this respondent thinks that both the mean earnings and the earnings inequality are too high.

Exploring further the results of the decomposition of JI1, we calculate the mean of the actual earnings amounts, obtaining $\$ 31,500$, and the mean of the just earnings amounts, obtaining $\$ 21,809$. This respondent indeed judges that the actual mean earnings is larger than the just mean earnings; we can confirm that the mean component of JI1, shown in Table 13, panel B, is: 


$$
\mathrm{JI} 1_{\text {Mean }}=\ln \left(\frac{31,500}{21,809}\right) \approx .368 .
$$

Similarly, exploring the inequality component of JI1, which is based on one of Atkinson's (1970, 1975 ) measures of inequality (the measure defined as one minus the ratio of the geometric mean to the arithmetic mean), we find that the Atkinson-inequality of actual earnings is .291 and of just earnings is .0363 . This respondent thus finds actual inequality much larger than just inequality; we can confirm that the inequality component of JI1, shown in Table 13, panel B, is:

$$
\mathrm{JI}_{\text {Ineq }}=\ln \left(\frac{1-.291}{1-.0363}\right) \approx-.307 .
$$

Of course, the estimates presented in Tables 12 and 13 become substantially more meaningful when they are compared across respondents. Moreover, groups and collectivities can then be characterized by the degree to which their members are similar to each other or exhibit agreement with respect to the justice quantities and relations. For example, it turns out that this respondent, whose justice life we have highlighted in our illustration, is similar to the overwhelming majority in judging that the actual inequality in the vignettes is too high. However, the hypothesis that the respondents can be described by the same just earnings function is soundly rejected, as is the hypothesis that the respondent-specific just earnings distributions are drawn from the same underlying distribution (Jasso unpubl).

\section{CONCLUDING NOTE}

This paper described procedures for measuring and estimating the fundamental quantities in justice analysis. We examined a variety of methods for measuring the actual reward and the just reward, for both self and other, including direct and indirect methods for measuring the just reward, and we provided an illustration of one of the two indirect methods, the one-reward-perrewardee method.

The justice evaluation function makes it possible to estimate the true just reward in the multiple-rewards-per-rewardee method, and the combination of the justice evaluation function 
and Rossi's factorial survey method makes it possible to estimate the true just reward in the onereward-per-rewardee method. This is a marriage of true minds, and its progeny include not only the true just reward measures but also measures of the experienced justice evaluation, as well as all the quantities and relations which depend on good measures of the experienced justice evaluation and the true just reward -- among them the just reward function, the principles of microjustice and macrojustice, the justice indexes, and the components of injustice due to poverty and to inequality.

The tasks ahead include systematic contrasts of all the methods collected here, in order to establish calibration factors by respondent and situational characteristics. 


\section{REFERENCES}

Atkinson, Anthony B. 1970. "On the Measurement of Inequality." Journal of Economic Theory 2:244-263.

. 1975. The Economics of Inequality. London: Oxford.

Berger, Joseph, Morris Zelditch, Jr., Bo Anderson, and Bernard P. Cohen. 1972. "Structural Aspects of Distributive Justice: A Status-Value Formulation." Pp. 119-246 in Joseph Berger, Morris Zelditch, and Bo Anderson (eds.), Sociological Theories in Progress, Volume 2. Boston: Houghton Mifflin.

Dar, Yechezkel, and Nura Resh. 1993. "Exploring the Multi-dimensional Structure of Deprivation Among Israeli Adolescents." Megamot 35:38-61. (In Hebrew). and . 1996. "Exploring the Persistence of Academic Achievement Gap: Social Differentials in Family Resource Returns in Israel.” Pp. 233-261 in Aaron Pallas (ed.), Research in Sociology of Education and Socialization, Vol. 11. Greenwich, CT: JAI Press.

Evans, M. D. R. 1989. "Distributive Justice: Some New Measures." Presented at the meeting of the International Sociological Association's Research Committee 28 on Social Stratification and Mobility, Stanford, California, August 1989.

Hagan, John, Gabrielle Ferrales, and Guillermina Jasso. Unpubl. "How Law Rules: Terror, Torture, and the Normative Judgments of Iraqi Judges.” Paper presented at the annual meeting of the American Sociological Association, Montreal, Canada, August 2006.

Homans, George Caspar. 1974. Social Behavior: its Elementary Forms. Rev. ed. New York: Harcourt, Brace, Jovanovich. 1976. "Commentary." Pp. 231-44 in Advances in Experimental Social Psychology, vol. 9, edited by Leonard Berkowitz and Elaine Walster. New York: Academic Press. Jasso, Guillermina. 1978. "On the Justice of Earnings: A New Specification of the Justice Evaluation Function." American Journal of Sociology 83:1398-1419. . 1980. "A New Theory of Distributive Justice." American Sociological Review 45:3- 
32.

. 1990. "Methods for the Theoretical and Empirical Analysis of Comparison Processes." Sociological Methodology 20:369-419.

. 1996. "Exploring the Reciprocal Relations between Theoretical and Empirical Work:

The Case of the Justice Evaluation Function (Paper in Honor of Robert K. Merton)." Sociological Methods and Research 24:253-303.

. 1999. "How Much Injustice Is There in the World? Two New Justice Indexes." American Sociological Review 64:133-168.

. 2003a. "Basic Research.” Pp. 52-53 in Michael Lewis-Beck, Alan Bryman, and Tim

Futing Liao (eds.), The Sage Encyclopedia of Social Science Research Methods, Volume

1. Thousand Oaks, CA: Sage Publications.

. 2003b. "Factorial Survey Method (Rossi's Method)." Pp. 374-376 in Michael Lewis-

Beck, Alan Bryman, and Tim Futing Liao (eds.), The Sage Encyclopedia of Social

Science Research Methods, Volume 1. Thousand Oaks, CA: Sage Publications. . 2006a. "Emotion in Justice Processes." Pp. 321-346 in Jan E. Stets and Jonathan H.

Turner (eds.), Handbook of the Sociology of Emotions. New York: Springer.

. 2006b. "Factorial Survey Methods for Studying Beliefs and Judgments." Sociological

Methods and Research 34:334-423.

, and Eva M. Meyersson Milgrom. Unpubl. "Perceptions of CEO Compensation.” Paper

presented at Stanford Workshop on Executive Compensation and Factorial Survey

Methods, June 2005.

, and Nura Resh. 2002. "Exploring the Sense of Justice about Grades." European

Sociological Review 18:333-351.

, and Peter H. Rossi. 1977. "Distributive Justice and Earned Income." American

Sociological Review 42:639-651.

, and Murray Webster 1999. "Assessing the Gender Gap in Just Earnings and Its

Underlying Mechanisms." Social Psychology Quarterly 62:367-380. 
, and Bernd Wegener. 1997. "Methods for Empirical Justice Analysis: Part I.

Framework, Models, and Quantities." Social Justice Research 10:393-430.

, and . 1999. "Gender and Country Differences in the Sense of Justice: Justice

Evaluation, Gender Earnings Gap, and Earnings Functions in Thirteen Countries."

International Journal of Comparative Sociology 40:94-116.

Kelley, Jonathan, and Mariah D. R. Evans. 1987. "IsssA: International Social Science Survey / Australia: Ideology of Inequality 1987-88: Questionnaire.” International Survey Centre and Research School of Social Sciences, Australia National University.

Rossi, Peter H. 1951. The Application of Latent Structure Analysis to the Study of Social

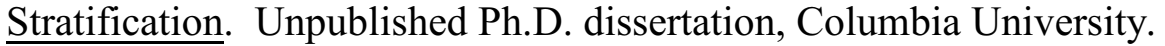
. 1979. "Vignette Analysis: Uncovering the Normative Structure of Complex Judgments." Pp. 176-186 in Qualitative and Quantitative Social Research: Papers in

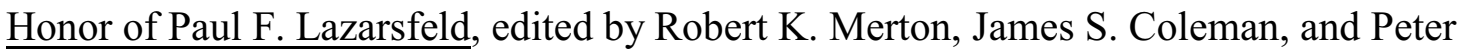
H. Rossi. New York: Free Press.

and Andy B. Anderson. 1982. "The Factorial Survey Approach: an Introduction." Pp. 15-67 in Measuring Social Judgments: the Factorial Survey Approach, edited by Peter H. Rossi and Steven L. Nock. Beverly Hills: Sage. and Richard A. Berk. 1985. "Varieties of Normative Consensus." American Sociological Review 50:333-347.

, William A. Sampson, Christine E. Bose, Guillermina Jasso, and Jeffrey Passel. 1974.

"Measuring Household Social Standing." Social Science Research 3:169-190.

Stevens, S. S. 1975. Psychophysics: an Introduction to Its Perceptual, Neural, and Social Prospects. Edited by Geraldine Stevens. New York: Wiley.

Wagner, David, and Joseph Berger. 1985. "Do Sociological Theories Grow?" American Journal of Sociology 90:697-728.

Weber, Max. 1958. The Protestant Ethic and the Spirit of Capitalism.

Translated by Talcott Parsons. New York: Scribner's. First published 
in 1904-1905.

Whitmeyer, Joseph M. 2004. "Past and Future Applications of Jasso's Justice Theory." Sociological Theory 13:432-444. 
Table 1. Observer-by-Rewardee Matrices of the Actual Reward, the Just Reward, and the Justice Evaluation

1. Actual Reward Matrix

$$
\boldsymbol{A}=\left[\begin{array}{ccccc}
a_{11} & a_{12} & a_{13} & \ldots & a_{1 R} \\
a_{21} & a_{22} & a_{23} & \ldots & a_{2 R} \\
a_{31} & a_{32} & a_{33} & \ldots & a_{3 R} \\
\vdots & \vdots & \vdots & \ddots & \vdots \\
a_{N 1} & a_{N 2} & a_{N 3} & \ldots & a_{N R}
\end{array}\right]
$$

If there are no perception errors, the actual reward matrix collapses to a vector:

$$
\boldsymbol{a}_{. \boldsymbol{r}}=\left[\begin{array}{lllll}
a_{.1} & a_{.2} & a_{.3} & \ldots & a_{. R}
\end{array}\right]
$$

\section{Just Reward Matrix}

$$
\boldsymbol{C}=\left[\begin{array}{ccccc}
c_{11} & c_{12} & c_{13} & \ldots & c_{1 R} \\
c_{21} & c_{22} & c_{23} & \ldots & c_{2 R} \\
c_{31} & c_{32} & c_{33} & \ldots & c_{3 R} \\
\vdots & \vdots & \vdots & \ddots & \vdots \\
c_{N 1} & c_{N 2} & c_{N 3} & \ldots & c_{N R}
\end{array}\right]
$$

\section{Justice Evaluation Matrix}

$$
\boldsymbol{J}=\left[\begin{array}{ccccc}
j_{11} & j_{12} & j_{13} & \ldots & j_{1 R} \\
j_{21} & j_{22} & j_{23} & \ldots & j_{2 R} \\
j_{31} & j_{32} & j_{33} & \ldots & j_{3 R} \\
\vdots & \vdots & \vdots & \ddots & \vdots \\
j_{N 1} & j_{N 2} & j_{N 3} & \ldots & j_{N R}
\end{array}\right]
$$

Notes: Observers are indexed by $\boldsymbol{o}=1, \ldots, N$; rewardees are indexed by $r=1, \ldots, R$.

Thus, $c_{o r}, a_{o r}, j_{o r}$ represent the observer-specific/rewardee-specific just reward, actual reward, and justice evaluation, respectively. 
Table 2. Occupations Studied in ISSP, ISJP, NIS, and GSOEP

\begin{tabular}{|c|c|c|c|c|c|c|}
\hline \multirow{2}{*}{ Occupation } & \multicolumn{3}{|c|}{ ISSP } & \multirow{2}{*}{$\begin{array}{c}\text { ISJP } \\
1991-2006 \\
\end{array}$} & \multirow{2}{*}{$\begin{array}{c}\text { NIS } \\
\text { NIS-2003-1 } \\
\end{array}$} & \multirow{2}{*}{$\begin{array}{c}\text { GSOEP } \\
2005 \\
\end{array}$} \\
\hline & 1987 & 1992 & 1999 & & & \\
\hline unskilled worker in a factory & $\checkmark$ & $\checkmark$ & $\checkmark$ & $\checkmark$ & $\checkmark$ & $\checkmark$ \\
\hline bricklayer & $\checkmark$ & $\checkmark$ & & & $\checkmark$ & \\
\hline farm laborer & $\checkmark$ & $\checkmark$ & & & $\checkmark$ & \\
\hline city bus driver & $\checkmark$ & $\checkmark$ & & & & \\
\hline doctor in general practice & $\checkmark$ & $\checkmark$ & $\checkmark$ & & $\checkmark$ & \\
\hline bank clerk & $\checkmark$ & $\checkmark$ & & & $\checkmark$ & \\
\hline owner of a small shop & $\checkmark$ & $\checkmark$ & & & $\checkmark$ & \\
\hline chairman of a large natl corp & $\checkmark$ & $\checkmark$ & $\checkmark$ & $\checkmark$ & $\checkmark$ & $\checkmark$ \\
\hline skilled worker in a factory & $\checkmark$ & $\checkmark$ & $\checkmark$ & & $\checkmark$ & \\
\hline secretary & $\checkmark$ & $\checkmark$ & & & $\checkmark$ & \\
\hline member of the cab in fed govt & $\checkmark$ & $\checkmark$ & $\checkmark$ & & & \\
\hline secondary school teacher & & & & & $\checkmark$ & \\
\hline engineer & & & & & $\checkmark$ & \\
\hline college professor & & & & & $\checkmark$ & \\
\hline shop assistant & & & $\checkmark$ & & & \\
\hline owner-manager of large factory & & & $\checkmark$ & & & \\
\hline judge in high court & & & $\checkmark$ & & & \\
\hline lawyer & & & $\checkmark$ & & & \\
\hline
\end{tabular}

Note: The wordings differ somewhat for the GSOEP occupations; see text. 
Table 3. Characteristics of Fictitious CEOs, Salary Perceptions Study

\section{Age}

Eleven levels, in increments of five years, from 20 to 70 years.

2. Sex

(1) Male

(2) Female

\section{Years of Schooling Completed}

Fifteen levels, in increments of one year, from completion of sixth grade to a doctoral degree.

\section{Years as CEO}

Sixteen levels, in increments of one year, from 0 to 15 years.

\section{Firm Headquarters}

(1) United States

(2) Europe

(3) Asia

\section{Industry of This Firm}
(1) Manufacturing
(2) Finance and insurance
(3) Information
(4) Wholesale trade

\section{Size of Firm - Capitalization}

Twenty-seven levels, from $\$ 50$ million to $\$ 600$ billion. $[50 \mathrm{~m}, 75 \mathrm{~m}, 100 \mathrm{~m}, 125 \mathrm{~m}, 150 \mathrm{~m}, 175 \mathrm{~m}, 200 \mathrm{~m}, 250 \mathrm{~m}, 500 \mathrm{~m}, 600 \mathrm{~m}, 700 \mathrm{~m}, 800 \mathrm{~m}, 900 \mathrm{~m}$, $1 \mathrm{~b}, 5 \mathrm{~b}, 10 \mathrm{~b}, 15 \mathrm{~b}, 20 \mathrm{~b}, 25 \mathrm{~b}, 50 \mathrm{~b}, 75 \mathrm{~b}, 100 \mathrm{~b}, 200 \mathrm{~b}, 300 \mathrm{~b}, 400 \mathrm{~b}, 500 \mathrm{~b}, 600 \mathrm{~b}]$

Note: The population of fictitious CEOs (called "vignettes") consists of all the logically possible combinations of characteristics. Logically impossible combinations are deleted. These are defined as meeting one of the following conditions: (i) age minus schooling LT 5; and (ii) age minus years as CEO LT 16. Random samples are drawn from the adjusted population for presentation to respondents. 
Table 4. Instructions in CEO Salary Perceptions Study

Facsimile:

\section{SURVEY OF PERCEPTIONS OF CEO SALARIES}

To the Respondent:

Chief executive officers (CEOs) and their firms differ in a lot of ways. We have made up descriptions of different kinds of CEOs and firms. The firms' market value is expressed in U.S. dollars (note that a billion corresponds to what in Europe is called a milliard). All the CEOs are newly hired at the firms. Some have been a CEO before at other firms. We would like to know what you think is that CEO's total compensation for the first year. This total compensation amount includes salary, signing bonus (if any), value of restricted stock, savings and thrift plans, and other benefits, but excludes stock options. The total compensation amount is expressed in U.S. dollars.

When you read each description of a CEO, please write the dollar amount which best represents what you think is that CEO's total compensation for the first year.

You may change any of the amounts.

Your responses are completely confidential.

Thank you very much for your participation. 
Table 5. Example of CEO Vignette in Salary Perceptions Study

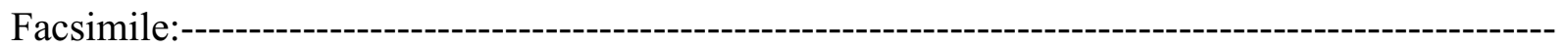

The CEO is 30 years old,

a man who completed 10 years of school.

He was a CEO elsewhere for 2 years.

The firm, headquartered in the United States,

is in the information sector.

The firm has a market value of \$ 300 billion.

ACTUAL TOTAL COMPENSATION 


\section{SURVEY OF JUDGMENTS ON THE JUSTICE OF EARNINGS}

To the Respondent:

People and their jobs differ in a lot of ways. We have made up descriptions of different kinds of people and jobs. All the persons described work full-time; and all have worked continuously and full-time since finishing school. Each person is randomly assigned a hypothetical earnings amount. We would like to know what you think about whether each person is fairly or unfairly paid, and, if you think that a person is unfairly paid, whether you think the person is paid too much or too little.

We would like you to use numbers to represent your judgments. Let zero represent the point of perfect justice. Let negative numbers represent degrees of underreward, and positive numbers represent degrees of overreward. The greater the degree of underpayment, the larger the absolute value of the negative number you choose (for example, if two persons receive ratings of -68 and -23 , the person receiving the -68 is viewed as more underpaid than the person receiving the -23). Similarly, the greater the degree of overpayment, the larger the positive number (for example, a person receiving a rating of +200 is viewed as more overpaid than a person receiving a rating of +75). In other words, mild degrees of underreward and of overreward are represented by numbers relatively close to zero; larger degrees of underreward and of overreward are represented by numbers farther away from zero.

The justice evaluation scale may be visualized as follows:

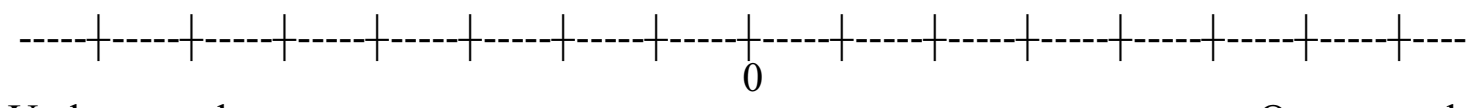

Underreward

Overreward

When you read each description of a person, please write the number that best matches your judgment about the fairness or unfairness of that person's earnings. There is no limit to the range of numbers that you may use. For example, some respondents like to map their personal scale to the numbers from -100 to +100 ; others prefer to use smaller regions, and still others, larger regions. Of course, you may choose any real number (for example, decimals and fractions as well as whole numbers) to represent a judgment.

You may rate the descriptions in any order.

You may change any of your ratings.

Your responses are completely confidential.

Thank you very much for your participation. 
Table 7. Facsimile of Vignette in Study of Justice of Earnings, Fall 2002

\begin{tabular}{l}
\hline \hline \\
A MAN 35 YEARS OLD, \\
WHO COMPLETED 12 YEARS OF SCHOOL, \\
GRADUATING FROM HIGH SCHOOL. \\
HE IS A WAREHOUSE SUPERVISOR. \\
HIS YEARLY SALARY IS \$37,500. \\
YOUR RATING \\
\hline \hline
\end{tabular}




\section{Table 8. Facsimile of Rating Instructions in Multiple-Rewards-Per-Rewardee Study of}

Justice of Earnings

\section{SURVEY OF JUDGMENTS ON THE JUSTICE OF EARNINGS}

To the Respondent:

People and their jobs differ in a lot of ways. We have made up descriptions of different kinds of people and jobs. All the persons described work full-time; and all have worked continuously and full-time since finishing school. Each person is randomly assigned several hypothetical earnings amounts. We would like to know what you think about whether, at each earnings amount, each person is fairly or unfairly paid, and, if you think that a person is unfairly paid, whether you think the person is paid too much or too little.

We would like you to use numbers to represent your judgments. Let zero represent the point of perfect justice. Let negative numbers represent degrees of underreward, and positive numbers represent degrees of overreward. The greater the degree of underpayment, the larger the absolute value of the negative number you choose (for example, if two earnings amounts receive ratings of -68 and -23 , the earnings amount receiving the -68 is viewed as greater underpayment than the earnings amount receiving the -23). Similarly, the greater the degree of overpayment, the larger the positive number (for example, an earnings amount receiving a rating of +200 is viewed as greater overpayment than an earnings amount receiving a rating of +75 ). In other words, mild degrees of underreward and of overreward are represented by numbers relatively close to zero; larger degrees of underreward and of overreward are represented by numbers farther away from zero.

The justice evaluation scale may be visualized as follows:

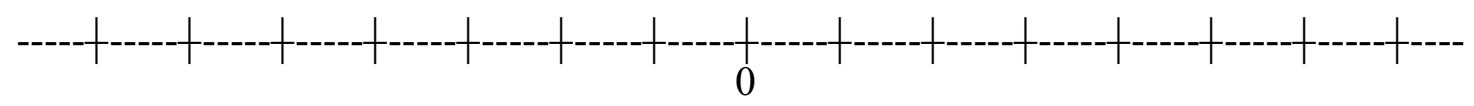

Underreward

Overreward

When you read each description of a person and an earnings amount, please write the number that best matches your judgment about the fairness or unfairness of that earnings for that person. There is no limit to the range of numbers that you may use. For example, some respondents like to map their personal scale to the numbers from -100 to +100 ; others prefer to use smaller regions, and still others, larger regions. Of course, you may choose any real number (for example, decimals and fractions as well as whole numbers) to represent a judgment.

You may change any of your ratings.

Your responses are completely confidential.

Thank you very much for your participation. 
Table 9. Facsimile of Vignette in Multiple-Reward-Per-Rewardee Study of Justice of Earnings

\author{
A MAN 43 YEARS OLD, \\ WHO COMPLETED 16 YEARS OF SCHOOL, \\ GRADUATING FROM COLLEGE WITH A B.A. DEGREE. \\ HE IS A GRADE SCHOOL TEACHER.
}

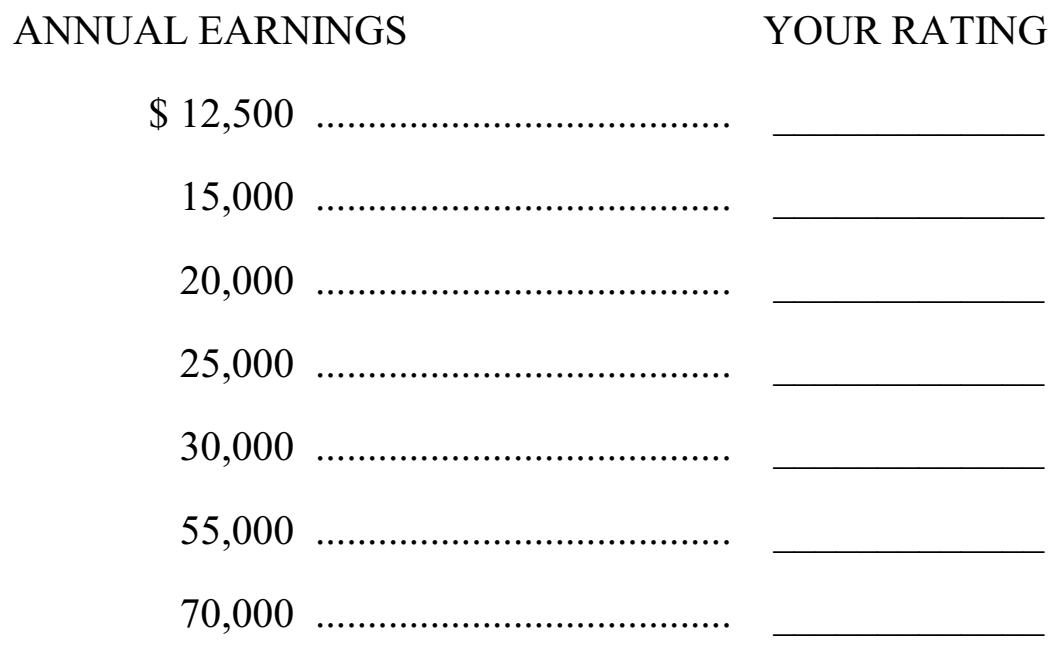




\section{Table 10. Facsimile of Instructions in Study of the Justice of Earnings, Using the One- Reward-Per-Rewardee Design}

\section{SURVEY OF JUDGMENTS ON THE JUSTICE OF EARNINGS}

To the Respondent:

People and their jobs differ in a lot of ways. We have made up descriptions of different kinds of people and jobs. All the persons described work full-time; and all have worked continuously and full-time since finishing school. Each person is randomly assigned a hypothetical earnings amount. We would like to know what you think about whether each person is fairly or unfairly paid, and, if you think that a person is unfairly paid, whether you think the person is paid too much or too little.

We would like you to use numbers to represent your judgments. Let zero represent the point of perfect justice. Let negative numbers represent degrees of underreward, and positive numbers represent degrees of overreward. The greater the degree of underpayment, the larger the absolute value of the negative number you choose (for example, if two persons receive ratings of -68 and -23 , the person receiving the -68 is viewed as more underpaid than the person receiving the -23). Similarly, the greater the degree of overpayment, the larger the positive number (for example, a person receiving a rating of +200 is viewed as more overpaid than a person receiving a rating of +75 ). In other words, mild degrees of underreward and of overreward are represented by numbers relatively close to zero; larger degrees of underreward and of overreward are represented by numbers farther away from zero.

The justice evaluation scale may be visualized as follows:

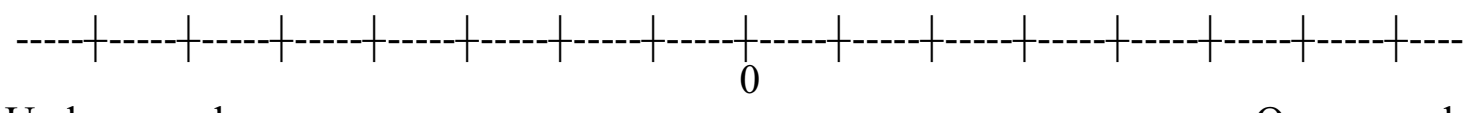

Underreward

Overreward

When you read each description of a person, please write the number that best matches your judgment about the fairness or unfairness of that person's earnings. There is no limit to the range of numbers that you may use. For example, some respondents like to map their personal scale to the numbers from -100 to +100 ; others prefer to use smaller regions, and still others, larger regions. Of course, you may choose any real number (for example, decimals and fractions as well as whole numbers) to represent a judgment.

You may rate the descriptions in any order.

You may change any of your ratings.

Your responses are completely confidential.

Thank you very much for your participation. 
Table 11. Facsimile of Vignette in Study of the Justice of Earnings, Using the One-RewardPer-Rewardee Design

\begin{tabular}{l}
\hline \hline A WOMAN 35 YEARS OLD, \\
WHO COMPLETED 12 YEARS OF SCHOOL, \\
GRADUATING FROM HIGH SCHOOL. \\
SHE IS A WAREHOUSE SUPERVISOR. \\
HER YEARLY SALARY IS \$37,500. \\
YOUR RATING_ \\
\hline \hline
\end{tabular}


Table 12. Measurement/Estimation in Empirical Justice Analysis: Data and Estimates of the Expressed Justice Evaluation Equation, the Experienced Justice Evaluation, and the True Just Reward, for One Respondent

\begin{tabular}{|c|c|c|c|c|}
\hline \multirow[t]{2}{*}{ Worker } & \multicolumn{2}{|c|}{ Raw Data } & \multicolumn{2}{|c|}{ Estimates } \\
\hline & $\begin{array}{c}\text { Actual } \\
\text { Earnings } \\
(1999 \$)\end{array}$ & $\begin{array}{c}\text { Expressed } \\
\text { Justice } \\
\text { Evaluation }\end{array}$ & $\begin{array}{c}\text { Experienced } \\
\text { Justice } \\
\text { Evaluation }\end{array}$ & $\begin{array}{c}\text { True } \\
\text { Just Earnings } \\
(1999 \$)\end{array}$ \\
\hline 1 & 25,000 & 2.5 & .433 & 16,211 \\
\hline 2 & 5,000 & -10 & -1.73 & 28,281 \\
\hline 3 & 30,000 & 2.5 & .433 & 19,453 \\
\hline 4 & 7,500 & -9 & -1.56 & 35,673 \\
\hline 5 & 15,000 & -1 & -.173 & 17,838 \\
\hline 6 & 35,000 & 1 & .173 & 29,432 \\
\hline 7 & 35,000 & 2.5 & .433 & 22,695 \\
\hline 8 & 25,000 & 0 & 0 & 25,000 \\
\hline 9 & 5,000 & -8.5 & -1.47 & 21,808 \\
\hline 10 & 55,000 & 7.5 & 1.30 & 14,996 \\
\hline 11 & 7,500 & -2.5 & -.433 & 11,566 \\
\hline 12 & 10,000 & -3 & -.520 & 16,817 \\
\hline 13 & 45,000 & 3 & .520 & 26,758 \\
\hline 14 & 15,000 & 0 & 0 & 15,000 \\
\hline 15 & 55,000 & 4 & .693 & 27,501 \\
\hline 16 & 10,000 & -4 & -.693 & 19,999 \\
\hline 17 & 100,000 & 10 & 1.73 & 17,679 \\
\hline 18 & 55,000 & 5 & .866 & 23,126 \\
\hline 19 & 70,000 & 7.5 & 1.30 & 19,085 \\
\hline 20 & 25,000 & -.5 & -.0866 & 27,263 \\
\hline
\end{tabular}

B. Estimated Expressed Justice Evaluation Equation

$$
\text { intercept }=-57.4 \quad \text { signature constant }=5.77 \quad R^{2}=.912
$$

Note: Data are drawn from a larger study reported in Jasso (unpubl). Workers \#1-\#5 and \#16\#20 are women, the rest men. 
Table 13. Measurement/Estimation in Empirical Justice Analysis: Estimates of Additional Justice Quantities -- the Principles of Microjustice and Macrojustice, the Just Gender Wage Gap, and the Justice Indexes -- for One Respondent

A. Quantities Pertaining to the First Central Question:

What Do Individuals and Societies Think Is Just, and Why?

1. Principles of Microjustice

just base wage

$\$ 10,207$

just rate of return to schooling

.0562

just gender multiplier

1.05

$R^{2}$ of just earnings function

2. Principles of Macrojustice

just Gini's inequality

just Atkinson's inequality

just Plato's ratio

just relative minimum

just relative maximum

3. Just Gender Wage Gaps

mean-based female/male ratio

median-based female/male ratio

.886

B. Quantities Pertaining to the Third Central Question:

What Is the Magnitude of the Perceived Injustice Associated with Given Departures from Perfect Justice?

1. Qualitative Justice Evaluation

percent workers unjustly underpaid

percent workers justly paid

percent workers unjustly overpaid

2. Justice Indexes

JI1 .0606

mean component of JI1 .368

inequality component of JI1 $-.307$

J12 .728

Note: Data are drawn from a larger study reported in Jasso (unpubl). 